\title{
Risk of premature birth in multifetal pregnancy
}

\author{
H Minakami, S Kosuge, H Fujiwara, Y Mori and I Sato \\ Center for Perinatal Medicine, Jichi Medical School Hospital, Minamikawachi-machi, Tochigi, 329-04 Japan
}

\begin{abstract}
The risk of preterm delivery ( $<37$ weeks of gestation) is approximately nine times higher in women with multifetal pregnancies than in women with singleton pregnancies. However, it is possible that the risk will vary according to gestational week. To assess the risk of premature birth within 1 week by gestational age among multifetal pregnancies and compare the estimated risk with that of singleton pregnancies, we analyzed 6036475 infants born in singleton pregnancies and 90887 infants born in multifetal pregnancies in Japan ( $\geq 22$ weeks) over the 5-year period 1989-1993. An estimate of the risk of birth within 1 week at gestational week $n$ was obtained by dividing the number of infants delivered at gestational week $n$ by the number of infants delivered at or beyond gestational week $\mathrm{n}$. The risk at 22 weeks was 0.9 per 1000 fetuses for singleton pregnancies and 5.0 per 1000 for multifetal pregnancies. The risk remained relatively stable until 27 weeks of gestation, then sharply increased toward 36 weeks of gestation in both singleton and multifetal pregnancies. The odds ratio for birth within 1 week for fetuses of multifetal pregnancies compared with fetuses of singl eton pregnancies was $5.9(95 \% \mathrm{Cl}, 5.4-6.5)$ at 22 week s of gestation, increasing gradually with increasing gestational age until 33 weeks of gestation $(13.7 ; 95 \% \mathrm{Cl}$, $13.1-14.2)$ but declining ther eafter to $8.8(95 \% \mathrm{Cl}, 8.6-8.9)$ at 36 week s of gestation. Results of data analysis for each year of the 5-year period did not differ substantially. Twin Research (2000) 3, 2-6.
\end{abstract}

Keywords: low birth weight infant, national statistics, premature labor, triplets, twins

\section{Introduction}

The increasing use of assisted reproductive techniques over the past decade has led to an increase in the number of multifetal pregnancies. ${ }^{1,2}$ The perinatal mortality rate is approximately five times higher among twins than among infants of singleton pregnancies. ${ }^{3}$ This high rate is mainly attributed to premature birth, with the risk of premature delivery being approximately nine times higher in women with multifetal pregnancies than in women with singleton pregnancies: $38 \%$ of women with multifetal pregnancies give birth before 37 weeks of gestation, compared with $4.2 \%$ of women with singleton pregnancies. ${ }^{3}$ Thus, women with multifetal pregnancies are at a substantially higher risk of premature birth than women with singleton pregnancies. However, the risk of giving birth within 1 week in women with multifetal pregnancies at a given gestational week has not been evaluated. It is possible that this risk will vary according to gestational week and depend on the gestational week. We therefore assessed the risk of giving birth pre-

Correspondence: Hisanori Minakami, MD, Center for Perinatal Medicine, Jichi Medical School Hospital, Minamikawachi-machi, Tochigi, 329-0498 Japan. Tel: +81285442111; Fax: + 8128544 8505; E-mail: minasho@ichi.ac.jp

Received 4 December 1998; revised 5 January 1999; accepted 26 October 1999 maturely within 1 week by gestational age using Japanese national statistics, ${ }^{4}$ comparing the relative risk in multifetal versus singleton pregnancies.

\section{Materials and methods}

We analyzed the data collected by the Japanese Ministry of Health and Welfare on all births (live and stillborn) that occurred in Japan at 22 weeks or more of gestation over the 5-year period between 1989 and 1993. Of the 6127362 infants analyzed, 90887 $(1.5 \%)$ were born to women with multifetal pregnancies (Table 1). The data did not include the number of fetuses in such multifetal pregnancies. However, approximately $96 \%$ of infants of multifetal pregnancies were born to mothers with twin pregnancies. ${ }^{2}$ Because an early examination by ultrasonography is widely practised in Japan, gestational age has been estimated by ultrasonography in most pregnancies. Infants of unspecified gestational age accounted for less than $0.04 \%$ of the total singleton and multifetal births and were excluded from the analysis.

Since the population at risk includes all undelivered fetuses, the risk of premature birth should be determined by dividing the number of impending premature births by the total number of undelivered fetuses. The risk of premature birth within 1 week in 
Table 1 Number of infants by gestational age documented in Japan

\begin{tabular}{|c|c|c|c|c|c|c|c|c|c|c|}
\hline \multirow{2}{*}{$\begin{array}{l}\text { Gestational } \\
\text { age, week }\end{array}$} & \multicolumn{5}{|c|}{ Singleton pregnancies } & \multicolumn{5}{|c|}{ Multifetal pregnancies } \\
\hline & 1989 & 1990 & 1991 & 1992 & 1993 & 1989 & 1990 & 1991 & 1992 & 1993 \\
\hline 22 & 1286 & 1218 & 966 & 919 & 827 & 77 & 98 & 97 & 108 & 85 \\
\hline 23 & 1241 & 1116 & 904 & 834 & 770 & 91 & 120 & 99 & 105 & 105 \\
\hline 24 & 820 & 804 & 815 & 774 & 772 & 84 & 123 & 81 & 98 & 83 \\
\hline 25 & 857 & 768 & 778 & 752 & 776 & 109 & 102 & 138 & 84 & 84 \\
\hline 26 & 819 & 864 & 813 & 814 & 757 & 99 & 115 & 102 & 151 & 120 \\
\hline 27 & 926 & 970 & 880 & 869 & 824 & 119 & 138 & 106 & 146 & 117 \\
\hline 28 & 1070 & 1045 & 996 & 1036 & 992 & 120 & 158 & 189 & 164 & 163 \\
\hline 29 & 1135 & 1149 & 1070 & 1144 & 1163 & 185 & 157 & 166 & 224 & 215 \\
\hline 30 & 1430 & 1368 & 1383 & 1453 & 1417 & 192 & 211 & 208 & 251 & 257 \\
\hline 31 & 1662 & 1665 & 1602 & 1690 & 1568 & 244 & 274 & 288 & 294 & 332 \\
\hline 32 & 2149 & 2260 & 2142 & 2155 & 2074 & 362 & 419 & 375 & 413 & 433 \\
\hline 33 & 3188 & 3156 & 3161 & 2976 & 2826 & 524 & 527 & 600 & 537 & 662 \\
\hline 34 & 5204 & 4992 & 4960 & 5014 & 4755 & 734 & 818 & 881 & 880 & 924 \\
\hline 35 & 9820 & 9751 & 9549 & 9643 & 9322 & 1279 & 1198 & 1278 & 1456 & 1475 \\
\hline 36 & 24103 & 24413 & 24637 & 24785 & 23595 & 2149 & 2284 & 2352 & 2689 & 2807 \\
\hline$\geqq 37$ & 1182641 & 1157022 & 1158121 & 1142373 & 1123117 & 11168 & 10679 & 11013 & 11139 & 11356 \\
\hline Total & 1238351 & 1212561 & 1212777 & 1197231 & 1175555 & 17536 & 17421 & 17973 & 18739 & 19218 \\
\hline
\end{tabular}

undelivered fetuses at gestational week $n$ was estimated based on a modification of the method of Yudkin et $\mathrm{al}^{5}$ as follows: risk of birth within 1 week at gestational week $n=$ number of infants delivered at gestational week $n$ divided by the number of infants delivered at or beyond gestational week $n$.

The odds ratio was used to estimate the relative risk of birth within 1 week for the infants of multifetal compared with singleton pregnancies. Miettinen's method ${ }^{6}$ was used to compute the $95 \%$ confidence interval.

\section{Results}

The risk for birth within 1 week by gestational age, which was obtained from an analysis of the data for each year and the data including the total population, is shown in Figure1. The risk did not differ substantially within the 5 years in the singleton and multifetal pregnancies. The risk at 22 weeks was about 0.9 per 1000 singleton pregnancies, implying that 1 in 1111 women at 22 weeks will give birth within 1 week (Figure 1A). The corresponding risk for multifetal pregnancies was about 5 per 1000 fetuses, implying that 1 in 200 fetuses at 22 weeks of gestation will be born within 1 week (Figure1B). Thus, the risk was much higher for multifetal than for singleton pregnancies. This risk remained stable until 27 weeks, and thereafter increased sharply with increasing gestational age for singleton as well as multifetal pregnancies. At 29 weeks of gestation, more than 10 in 1000 fetuses had a risk of birth within 1 week in the multifetal pregnancies, whereas a similar risk was seen at 36 weeks in the singleton pregnancies.
The odds ratio for birth at $<37$ weeks of gestation for an infant of multifetal pregnancy was 8.64 (95\% $\mathrm{Cl}, 8.56-8.72)$ as calculated from data in Table 1. However, the odds ratio for birth within 1 week for a multifetal vs a singl eton pregnancy at 22 weeks was $5.9(95 \% \mathrm{Cl}, 5.4-6.5)$ (Figure 2). The odds ratio increased gradually with increasing gestational age until 33 w eeks of gestation (13.7; 95\% Cl, 13.1-14.2), and declined thereafter to $8.8(95 \% \mathrm{Cl}, 8.6-8.9)$ at 36 weeks of gestation. Infants of multifetal pregnancies were 10 times more likely to be delivered within 1 week than were those of singl eton pregnancies over the 8-week period between 28 and 36 weeks of gestation.

The odds ratio for birth within 1 week for infants of multifetal pregnancies as compared with singleton infants varied somew hat by year, mainly because of the limited number of premature births among multifetal pregnancies. However, for each year, the lowest relative risk was seen at 22 weeks, and gradually increased until 32 to 33 weeks, then declined sharply thereafter (data not shown).

\section{Discussion}

The present results showed that the risk of birth within 1 week was related to gestational age in singleton as well as multifetal pregnancies. The risk of birth within 1 week for offspring of multifetal pregnancies compared with singletons also depended on gestational age. Thus, although the reported odds ratio for giving birth prematurely (ie at $<37$ weeks of gestation) in a multifetal pregnancy is nearly 9 times that of a singleton pregnancy, ${ }^{3}$ the 

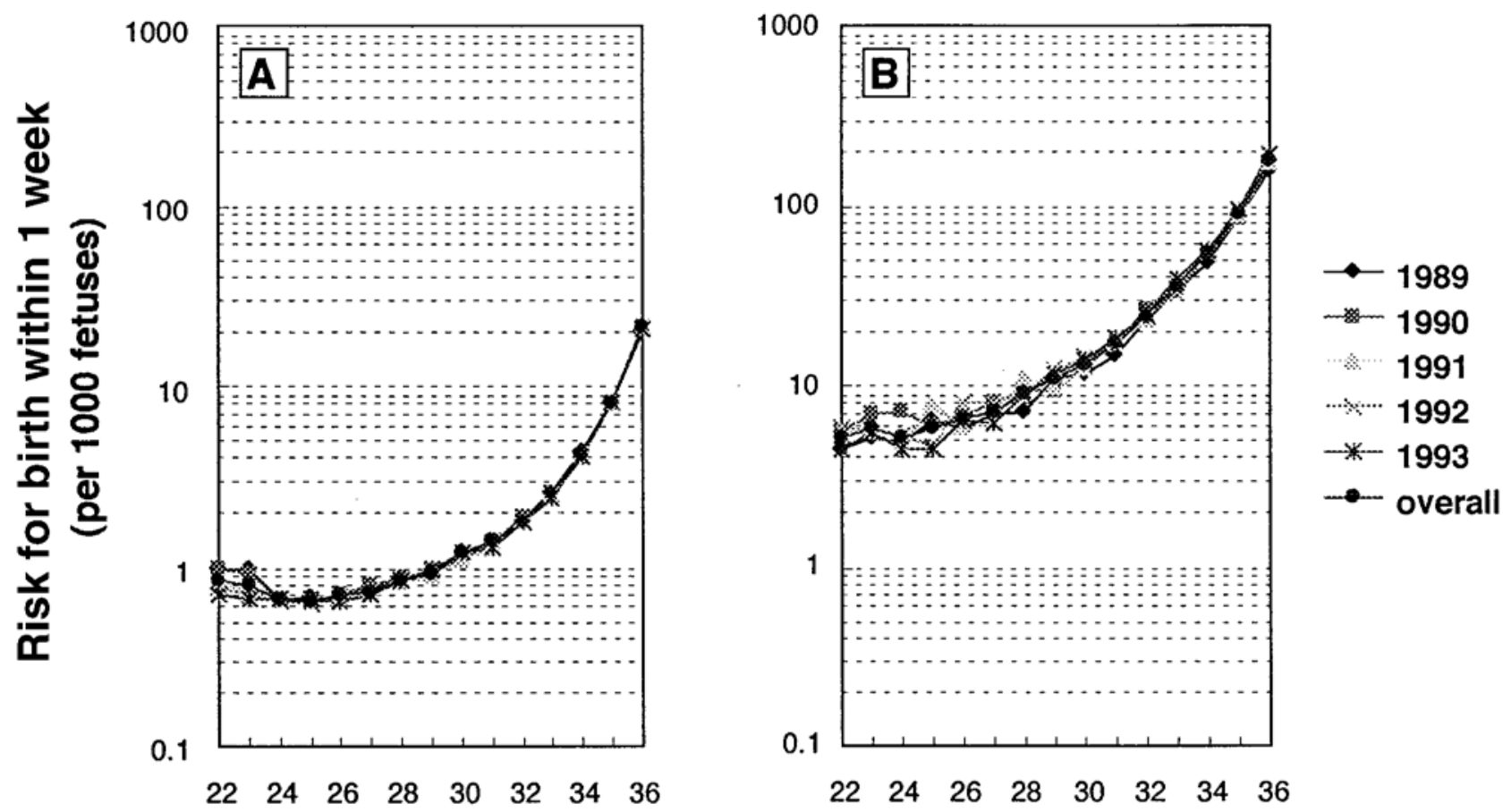

\section{Gestational week}

Figure 1 Risk for premature birth in each gestational week, A; singleton pregnancies, B; multiple pregnancies

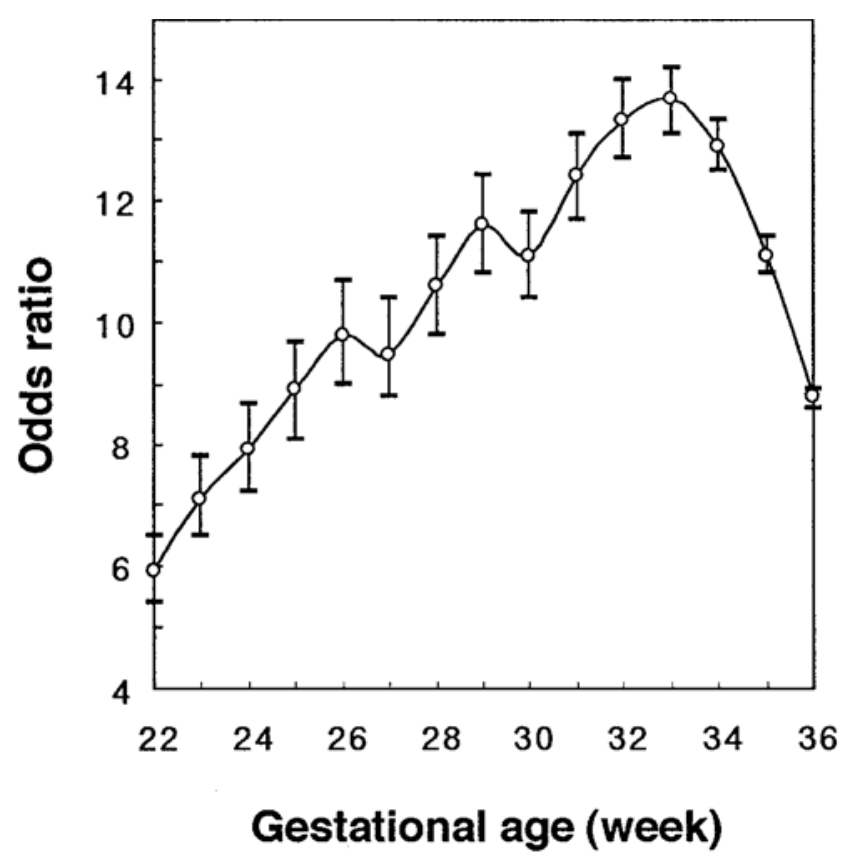

Figure 2 Odds ratio for premature birth with $95 \%$ confidence interval s (multiple pregnancy/singl eton pregnancy) in each gestational week, based on the total number of infants in Table 1 offspring of multifetal pregnancies of various gestational ages do not face an equal risk of premature birth within 1 week. The risk exceeded 10.0 over the 8 weeks between 28 and 36 weeks of gestation. This phenomenon may be important in developing measures to reduce the number of premature births in multifetal pregnancies.

Although the survival rate among premature infants has improved in recent years, a substantial number of such infants exhibit permanent disabilities such as cerebral palsy. ${ }^{7}$ Each year in Japan approximately 10000 newborns weigh less than $1500 \mathrm{~g}$ at birth; ${ }^{8}$ this group represents approximately $0.8 \%$ of all births. Of these, $65 \%$ are born alive, and about $55-60 \%$ of them survive ${ }^{8}$ because of major advances in neonatal intensive care. However, approximately $10 \%$ of the survivors develop cerebral palsy and $25-50 \%$ later show cognitive and behavioral deficiencies. ${ }^{9}$ Japanese infants of singleton pregnancies achieve a body weight of $1500 \mathrm{~g}$ at 30 weeks, ${ }^{10}$ whilst offspring of multifetal pregnancies achieve this weight at 31 weeks. ${ }^{11}$ In the present study, as many as $6.5 \%$ of the infants of multifetal pregnancies were born at $<31$ weeks of gestation compared with $0.63 \%$ of singleton infants born at $<30$ weeks. Thus, it can be expected that at least 
$1.25 \%$ of all infants born to multifetal pregnancies will survive, but with permanent disabilities: 0.065 (birth weight $<1500 \mathrm{~g}) \times 0.55$ (survivors) $\times 0.35$ (cerebral palsy and/or cognitive and behavioral deficiencies) $\times 100$. The corresponding number for singleton pregnancies is much lower, being $0.12 \%$ : 0.0063 (birth weight $<1500 \mathrm{~g}) \times 0.55$ (survivors) $\times 0.35$ (cerebral palsy and/or cognitive and behavioral deficiencies) $\times 100$. In our recent report of a cohort study of twin pregnancies managed by our group, ${ }^{12} 2.4 \%$ of infants died by 1 year of age, including intrauterine deaths, and $2.6 \%$ of infants exhibited permanent disabilities at 1 year of age. The number of infants with disabilities will increase with age, in that cognitive and behavioral dysfunctions become manifest over time. Thus, infants of multifetal pregnancies are at a higher risk of permanent disability than are infants of singleton pregnancies.

The reported increase in the incidence of multifetal pregnancies in Japan and other countries, ${ }^{1,2}$ which is also documented by the present study, is likely to lead to an increase in the number of children with disabilities. We found that infants of multifetal pregnancies that reached 28 weeks of gestation were 10 times more likely to be born within 1 week as compared with singleton infants of a corresponding gestational age. This finding emphasizes the need for women with multiple pregnancies to receive increased medical care. It al so points out the need to develop measures for detecting those women who are at an increased risk of giving birth prematurely. For instance, the estimation of the length of uterine cervix by ultrasonography in midtrimester reportedly detects those women at an increased risk of premature birth. ${ }^{13}$ Early medical intervention with a tocolytic agent, such as ritodrine, ${ }^{14}$ and antibiotic administration ${ }^{15,16}$ may help to extend gestation in those women who are at an increased risk of giving birth prematurely.

In the present study, approximately 1200 women had triplet or a higher order multifetal pregnancies, which accounted for approximately $4.0 \%$ of all infants born to the multifetal pregnancies. ${ }^{2}$ Thus our data may be applicable only to twin pregnancies. Because a shorter duration of pregnancy has been reported for triplets $\left(33.8\right.$ weeks) ${ }^{17}$ as well as for quadruplets (31.4 weeks), ${ }^{18}$ women with such pregnancies may be at a higher risk of giving birth within 1 week than those with twin pregnancies.

Some women with multifetal pregnancies are not concerned about the risk of preterm delivery, and may thus neglect the need for medical intervention, including care in hospital. However, it has not been demonstrated that prophylactic care in hospital or preventive measures are effective in reducing the number of premature births. ${ }^{1,20}$ The present data may be useful for counseling women with multifetal pregnancies, although the findings may apply only to twin pregnancies.

\section{Acknowledgements}

The authors are grateful to the Japanese Ministry of Health and Welfare for financial support (Pediatric Research Grant No. 10-02).

\section{References}

1 Petterson B, Nelson KB, Watson L, Stanley F. Twins, triplets, and cerebral palsy in births in Western Australia in the 1980s. BMJ 1993; 307: 1239-1243.

2 Imaizumi Y. Recent and long term trends of multiple birth rates and influencing factors in Japan. J Epidemiol 1994; 4: 103-109.

3 Minakami H, Sato I. Reestimating date of delivery in multifetal pregnancies. JAMA 1996; 275: 1432-1434.

4 Division of Maternal and Child Health, Children and Families Bureau. Annual Report on Maternal and Child Health Statistics in Japan. Ministry of Health and Welfare, Japan. 1990, 1991, 1992, 1993, 1994.

5 Yudkin PL, Wood L, Redman CWG. Risk of unexplained stillbirth at different gestational ages. Lancet 1987; i: 1192-1194.

6 Miettinen OS. Estimability and estimation in case-referent studies. Am J Epidemiol 1976; 103: 226-235.

7 Kuban KCK, Leviton A. Cerebral palsy. N Engl J Med 1994; 330: 188-195.

8 Minakami $\mathrm{H}$, Sato I. Birth-weight-specific perinatal mortality in Japan, 1989-1993: singleton vs multifetal pregnancies. Gynecol Obstet Inves 1999; 48: 38-42.

9 Volpe $J$. Neurologic outcome of prematurity. Arch Neurol 1998; 55: 297-300.

10 Nishida H, Sakanoue M, Kurachi K, Asada A, Kubo S, Funakawa H. Fetal growth curve of Japanese. Nippon Shinseiji Gakkai Zasshi 1984; 20: 90-97.

11 Minakami H, Izumi A, Sato I. Gestational age-specific normal birth weight for Japanese twins: risk of early neonatal death in small-for-gestational age and large-for-gestational age twins. J Reprod Med 1999; 44: 625-629.

12 Minakami H, Sayama M, Honma Y, Matsubara S, Koike T, Sato I, Uchida A, Eguchi Y, Momoi M, Araki S. Lower risks of adverse outcome in twins conceived by artificial reproductive techniques compared with spontaneously conceived twins. Hum Reprod 1998; 13: 2005-2008.

13 lams D, Goldenberg RL, Meis PJ, Mercer BM, Moawad A, Das A, Thom E, McNellis D, Copper RL, Johnson F, Roberts M. The length of the cervix and the risk of spontaneous premature delivery. N Engl J Med 1996; 334: 567-572.

14 Keirse M JNC. New perspectives for the effective treatment of preterm labor. Am J Obstet Gynecol 1995; 173: 618-628.

15 McGregor JA, French J, Parker R, Draper D, Patterson E, Jones $\mathrm{W}$, Thorsgard K, McFee J. Prevention of premature birth by screening and treatment for common genital tract infections: results of a prospective controlled evaluation. Am J Obstet Gynecol 1995; 173: 157-167. 
16 Hauth JC, Goldenberg RL, Andrews WW, DuBard MB, Copper $\mathrm{RL}$. Reduced incidence of preterm delivery with metronidazole and erythromycin in women with bacterial vaginosis. $\mathrm{N}$ Engl J Med 1995; 333: 1732-1736.

17 Elster AD, Bleyl $\mathbf{L}$, Craven TE. Birth weight standards of triplets under modern obstetric care in the United States, 1984-1989. Obstet Gynecol 1991; 77: 387-393.

18 Collins MS, Bleyl JA. Seventy-one quadruplet pregnancies: management and outcome. Am J Obstet Gynecol 1990; 162: 1384-1392.
19 MacLennan AH, Green RC, O'Shea R, Brookes C, Morris D. Routine hospital admission in twin pregnancy between 26 and 30 weeks' gestation. Lancet 1990; 335: 267-269.

20 Dyson DC, Danbe KH, Bamber JA, Crites YM, Field DR, Maier A, Newman LA, Ray DA, Walton DL, Armstrong MA. Monitoring women at risk for preterm labor. $\mathrm{N}$ Engl J Med 1998; 338: 15-19. 